\title{
Bicomponent hydrogen-bonded nanostructures formed by two complementary molecular Landers on $\mathrm{Au}(111)$
}

\author{
N. Kalashnyk, ${ }^{a}$ M. Yu, ${ }^{a}$ R. Barattin, ${ }^{b}$ Y. Benjalal, ${ }^{c}$ M. Hliwa,${ }^{b c}$ C. Joachim, ${ }^{b}$ E. Lægsgaard,${ }^{a}$ F. Besenbacher,${ }^{a}$ A. \\ Gourdon, ${ }^{b}$ X. Bouju ${ }^{* b}$ and T. R. Linderoth $*^{a}$
}

The co-adsorption of two molecular Landers equipped with functional groups capable of forming a complementary triple hydrogen-bonding motif is investigated with scanning tunneling microscopy and molecular mechanics calculations. Surprisingly, the anticipated complementary motif is not realised in 2-D terrace structures, but is observed in 1-D structures at step edges where molecular conformational flexibility is confined.

Molecular Landers are a class of molecules designed for surface-adsorption studies in which a poly-aromatic board is separated from the surface by bulky spacer groups acting as legs. ${ }^{1}$ The adsorption of molecular Landers on metal surfaces under ultra high vacuum conditions has been studied by scanning tunneling microscopy (STM), providing information regarding the organisation on terraces, ${ }^{2}$ step edges and templated surfaces $^{3}$ as well as the diffusional ${ }^{4,5}$ and electronic ${ }^{6,7}$ properties for these relatively large and complex molecules. A unique feature of molecular Landers is their ability to trap metal atoms and act as molecular moulds, ${ }^{8,9}$ potentially allowing for bottom-up organisation of metallic nanostructures through pre-assembly of molecular Landers. With the aim to steer the assembly of molecular Landers through hydrogen-bonding interactions, two linear and bi-dentate molecular Landers were recently synthesized ${ }^{10}$ each bearing either two diaminotriazine (DAT) or two di-carboxylic imide (DCI) functionalities (Fig. SI1). The DAT and DCI functionalities are complementary and can form a triple hydrogen bonding motif consisting of one $\mathrm{N}-\mathrm{H} \cdots \mathrm{N}$ and two $\mathrm{N}-\mathrm{H} \cdots \mathrm{O}$ bonds. This classical ${ }^{11,12}$ supra-molecular motif has on surfaces been observed to drive the organisation of both 2-D networks ${ }^{13,14}$ (involving molecules with 3 -fold symmetry) and 1-D chains ${ }^{15,16}$ (for linear molecules), but in all cases for planar molecules where the functional groups are adsorbed close to and parallel to the surface.

Here we investigate the co-adsorption of Lander DAT and Lander DCI on $\mathrm{Au}(111)$ where the molecular structure allows for more conformational flexibility. Surprisingly, our STM experiments reveal that on terraces of the Au(111) surface, the anticipated DAT-DCI motif is not borne out, but the linear Lander DCI and Lander DAT molecules instead form a 2-D network driven by a complex interaction node involving four molecules. The anticipated 1-D arrangement driven by complementary DCI-DAT interaction is instead realised when the molecules adsorb at step edges. The energetic and mechanistic reasons for these observations are identified from molecular mechanics calculations. Our results provide important insights into how the DAT-DCI motif may drive self-assembly on surfaces for complex molecules with large degrees of conformational flexibility.

The adsorption of Lander DCI and Lander DAT separately on Au(111) surfaces was investigated by us previously. ${ }^{17,18}$ Lander DCI forms 1$\mathrm{D}$ chains driven by double hydrogen bonding between the DCI groups, ${ }^{17}$ and Lander DAT forms various 2 -D networks in which the interaction between the DAT groups is optimised through conformational flexibility. ${ }^{18}$ In the co-deposition experiments reported upon here Lander DAT and Lander DCI were evaporated from separate crucibles onto a Au(111) surface followed by annealing to $400 \mathrm{~K}$. Details on experimental and theoretical methods are provided in the supplementary information section. Co-deposition of Lander DAT and DCI at submonolayer coverage results on the terraces of the $\mathrm{Au}(111)$ surface in the formation of 2D molecular islands with grid-like structure as shown in Fig. 1a. The STM contrast of Lander DAT and Lander DCI is dominated by the four peripheral leg groups which appear as bright protrusions (see Fig. SI1). The two molecules can therefore be distinguished based on analysis of the separation between the leg groups (Fig. SI2). In the 2D molecular island the Lander DCI molecules are arranged in chains via head-to-tail interaction and interspersed Lander DAT molecules separate the chains. The periodicity along the Lander DCI chains is $\sim 31.5 \AA$ similar to that determined for isolated 1D chains of Lander DCI molecules ${ }^{17}$ suggesting that the interaction between two neighbouring Lander DCI molecules in the $2 \mathrm{D}$ network involves the same double $\mathrm{N}-\mathrm{H} \cdots \mathrm{O}$ hydrogen bonding (dashed lines in Fig. 1a) as in the isolated 1D chains. From experimental images and the expected superimposed model, one estimates that the two $\mathrm{C}-\mathrm{O}$ of Lander DCI molecules belonging to adjacent chains (right panel of Fig. 1a) are separated by $\mathrm{d}(12)=\mathrm{d}\left(1^{\prime} 2^{\prime}\right)=26 \pm 0.9 \AA$, and accommodate well the size of interspersed Lander DAT molecules in this framework. Furthermore, the separation between the two C-O of Lander DCI molecules within the chains is $\mathrm{d}\left(11^{\prime}\right)=\mathrm{d}\left(22^{\prime}\right)=5.1 \pm 0.5 \AA$, which corresponds well to the distance between two N-H hydrogens in the DAT group of the Lander DAT molecule. As a consequence, each DAT Lander interacts with four DCI Landers via $\mathrm{N}-\mathrm{H}$-..O hydrogen bonding (solid lines in Fig. 1a). 

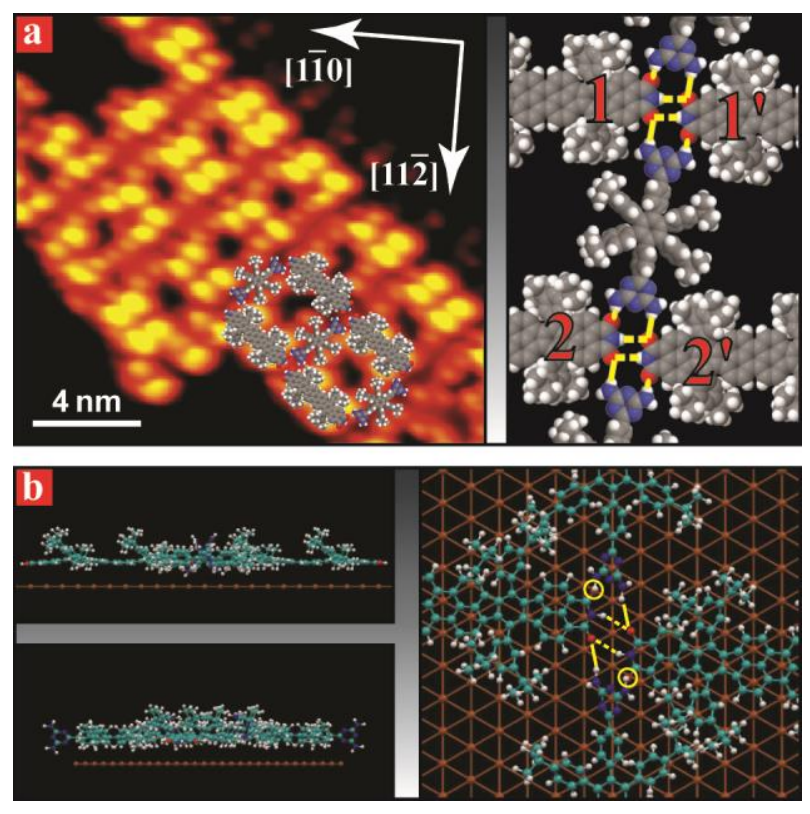

Fig. 1 Two-dimensional island formed from Lander DAT and DCI on the Au(111) surface. (a) STM image of 2D grid-like pattern (left panel) and a tentative model (right panel) of this structure. (b) Side (left panels) and top (right panel) views of the calculated model showing the Hbonding involved in formation of the $2 D$ network.

Molecular mechanics calculations of the 2D structure were performed focusing on a single interaction node composed of two Lander DCIs interconnected with two Lander DAT molecules. The fully relaxed structure is shown in Fig. $1 \mathrm{~b}$. The calculated distance $\mathrm{d}_{\mathrm{NHO}(1)}=2.51 \AA$ (dashed lines in Fig. 1b) corresponds well to the $\mathrm{N}-\mathrm{H} \cdots \mathrm{O}$ double hydrogen bonding between consecutive Lander DCI molecules in the chains, while the distances $\mathrm{d}_{\mathrm{NHO}(2)}=1.86 \AA$ and $\mathrm{d}_{\mathrm{NHO}(3)}=3.46 \AA$ (circles and solid lines in Fig. $1 \mathrm{~b}$, respectively) are assigned to the $\mathrm{H}$ bonding between two $\mathrm{N}-\mathrm{H}$ sites of the DAT group in Lander DAT and $\mathrm{C}-\mathrm{O}$ sites of two neighbouring DCI molecules in the chains. This interaction between Lander DAT and DCI molecules is additionally enabled by the ability of DAT groups to rotate and form H-bonding optimised in three dimensions as also previously found in structures formed from Lander DAT alone. ${ }^{18}$

The codeposition of Lander DAT and Lander DCI at very low coverage results in preferential anchoring of the molecules at step edges (Fig. 2a). Each molecule adsorbed at the step edge appears as two bright and two dim protrusions stemming from spacer legs standing on the upper and lower terraces, respectively, as also observed in previous STM studies on related Landers. ${ }^{19}$ The small and large four-lobed features have dimensions similar to those of Lander DAT and Lander DCI, respectively. Therefore, the short chains with periodicity $\sim 51.5 \AA$ are attributed to alternating Lander DAT and Lander DCI. Figure $2 \mathrm{~b}$ shows a calculated model for the 1D heteromolecular chain at the step edge, confirming this assignment. The calculated $\mathrm{N}-\mathrm{H} \cdots \mathrm{O}$ and $\mathrm{N}-\mathrm{H} \cdots \mathrm{N}$ distances (dashed and solid lines in Fig. 2b) between DAT and DCI groups within this structure are $2.3 \AA$ and $2.43 \AA$, respectively, and correspond well to the anticipated complementary triple H-bonding motif between the DAT and DCI functionalities.

Intriguingly, 1D hetero-molecular chains similar to those found at the step edges were never observed on the Au(111) terraces. To further illuminate the reasons for the preferential formation of 2D molecular networks on the terraces, we calculated the binding energies in the observed 2D network and a hypothetical 1D heteromolecular chain adsorbed on the terrace (Fig. 2c). The total energies and the contributions from different intermolecular interactions are summarised in Table SI1. The total energy per DAT-DCI molecular pair of the 2D structure is $1.51 \mathrm{eV}$, while that of the $1 \mathrm{D}$ chain at terrace is $0.44 \mathrm{eV}$. The H-bonding energy in the 2D structure contributes only $57 \%(0.86 \mathrm{eV})$ of this total energy as compared to $73 \%(0.32 \mathrm{eV})$ in the $1 \mathrm{D}$ case on terrace. Another reason for the higher energy gain in the 2D network is that the van der Waals interaction in this structure $(0.65 \mathrm{eV})$ is five times higher than the value calculated for the $1 \mathrm{D}$ chain on terraces $(0.12 \mathrm{eV})$. Therefore the van der Waals interaction is comparable to the H-bonding energy in the 2D network and imparts a large fraction of its stability. These calculations confirm that the formation of the $2 \mathrm{D}$ molecular network on terraces is more favourable than that of the $1 \mathrm{D}$ heteromolecular chains, in agreement with experimental observations. 


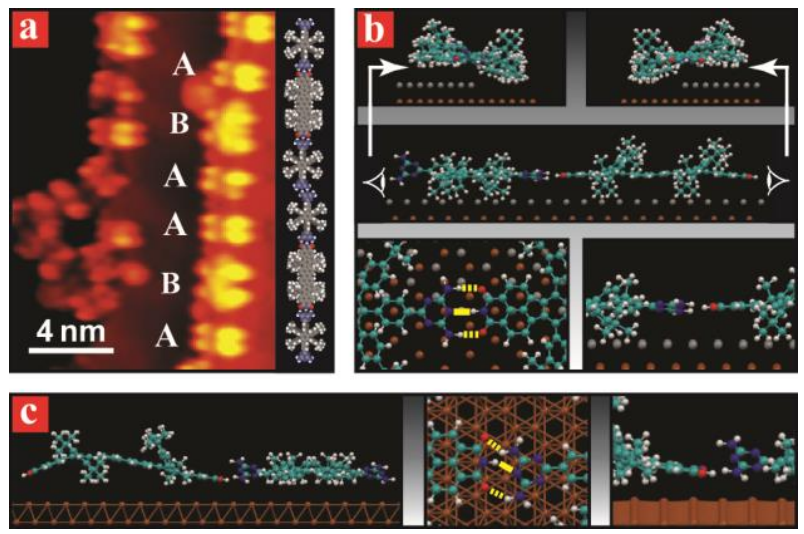

Fig. 2 One-dimensional structures formed from Lander DAT and DCI along the Au(111) step edges. (a) STM image of short molecular chains (left panel) composed of Lander DAT (A) and Lander DCI (B) and a tentative model (right panel) of this structure. (b) The calculated conformation of the $1 D$ chain at step edge: side view of this structure along (top panel) and perpendicular (middle panel) to the chain, and zoomed top (bottom-left panel) and side (bottom-right) views of the calculated model showing the H-bonding involved in formation of the $1 D$ structure. (c) The calculated model of a hypothetical 1D Lander DAT-DCI chain on terrace: side view (left panel) and zoomed top (middle panel) with side (right panel) views of the molecular conformations and interactions for Lander DAT and DCI molecules within this chain on terrace.

In the case of the 1D heteromolecular chain adsorbed at a step edge (Fig 2b) the total energy is 0.65 eV arising from van der Waals interaction $(0.05 \mathrm{eV})$ and $\mathrm{H}$-bonding $(0.59 \mathrm{eV})$. The hydrogen bonding between DCI and DAT molecules at step edge thus contributes more than $91 \%$ of the total energy. Therefore, owing to the presence of complementary end groups in both molecules, the triple H-bonding dominates over the van der Waals interaction, and drastically contributes to the formation of these short chains.

The calculated conformations and interactions for Lander DAT and Lander DCI molecules in a hypothetical 1D chain on the terrace are represented in Fig. 2c. The calculation shows that the Lander DCI molecule in a short DCI-DAT chain at step edge sits between the upper and the lower terrace with relatively straight adsorption geometry (Fig. 2b). In contrast, the backbone of the Lander DCI molecule adsorbed on the terrace is strongly distorted (Fig. 2c). The triple H-bonding between DCI and DAT molecules on the terrace will be strongly affected by the bent DCI conformation. At the step edge, the H-bonding between the complementary Lander molecules in the heteromolecular chain is further enhanced since the DAT groups are less tilted than on the terrace (Figs. 2b and 2c, respectively). As a result, the DAT-DCI hydrogen bonding in a chain is more directional and stronger at the step edge $(0.59 \mathrm{eV})$ than on the terrace $(0.32 \mathrm{eV})$ due to the adsorption conformations of the two interacting molecules. This is an important reason why the 1D heteromolecular chains are not observed experimentally on the terrace.

The scenarios for the formation of $1 \mathrm{D}$ and $2 \mathrm{D}$ structures are closely related to the ability of the DAT groups to rotate with respect to the central core of the Lander DAT molecule. The terrace promotes adsorption of Lander DAT with the poly-aromatic core parallel to the plane of the surface. This conformation induces a steric hindrance causing rotation of the DAT group. The DAT rotation leads to the formation of a 3-D optimized H-bonding with the DCI group of Lander DCI in the 2D network. The resulting rotated conformation of the DAT group is, however, not favourable for complementary DAT-DCI interaction in a 1D heteromolecular chain on the terrace. The consequence is thus twofold: i) the formation of a 2D network on the terrace consisting of Lander DCI chains interconnected by Lander DAT molecules and ii) the formation of heteromolecular chains at the step edge where the adsorption conformation straddling the step edge prevents rotation of the DAT group thereby allowing for DAT-DCI complementary interaction.

In summary we have investigated the codeposition of Lander DAT and DCI molecules on an Au(111) surface. At high coverage, these molecules self-assemble into an extended 2D grid-like structure on the terraces. This pattern appears to be composed of head-to-tail hydrogen-bonded Lander DCI chains interconnected through 3D optimized hydrogen bonding provided by Lander DAT molecules. At very low coverage, short 1D hetero-molecular chains built up with typical DAT-DCI-DAT sequential order are formed at step edges via complementary triple hydrogen bonds. Experimental and theoretical study shows that the multi-directional hydrogen bonding and van der Waals interaction between DCI-DCI and DAT-DCI groups involved in the 2D assembly contribute for the formation of stable molecular islands, whereas 1D short DAT-DCI-DAT chains are mainly stabilised at step edges via uni-directional triple hydrogen bonds. In other words, the step edge induces molecular conformations that favour the optimized formation of triple $\mathrm{H}$-bonds.

We acknowledge financial support from the EU programmes PICO-INSIDE, FRONTIERS and MONET as well as the Danish Natural Science Research Council. Part of this work was performed using High Performance Computing resources from the CALcul en MIdiPyrénées (CALMIP) facilities (Grant No. 2011-[P0832]). 


\section{Notes and references}

${ }^{a}$ Interdisciplinary Nanoscience Center (iNANO) and Department of Physics and Astronomy, Aarhus University, Aarhus 8000, Denmark.

E-mail: trolle@inano.au.dk

${ }^{b}$ Nanosciences group, CEMES-CNRS, UPR 8011, Bât. picoLab, BP 94347, F-31055 Toulouse, France.

E-mail : xavier.bouju@cemes.fr

${ }^{c}$ Faculté des Sciences Ben M’Sik, Université Hassan II-Mohammédia, Casablanca BP 7955, Morocco.

1 A. Gourdon, Eur. J. Org. Chem., 1998, 2797.

2 J. Kuntze, X. Ge and R. Berndt, Nanotechnology, 2004, 15, S337.

3 R. Otero, Y. Naitoh, F. Rosei, P. Jiang, P. Thostrup, A. Gourdon, E. Lægsgaard, I. Stensgaard, C. Joachim and F. Besenbacher, Angew. Chem. Int. Ed., 2004, 43, 2092.

4 R. Otero, F. Hummelink, F. Sato, S. B. Legoas, P. Thostrup, E. Lægsgaard, I. Stensgaard, D. S. Galvao and F. Besenbacher, Nat. Mater., 2004, 3, 779.

5 M. Schunack, T. R. Linderoth, F. Rosei, E. Lægsgaard, I. Stensgaard and F. Besenbacher, Phys. Rev. Lett., $2002,88,156102$.

6 L. Grill, K. H. Rieder, F. Moresco, S. Stojkovic, A. Gourdon and C. Joachim, Nano Lett., 2006, $6,2685$.

7 L. Grill, K. H. Rieder, F. Moresco, S. Stojkovic, A. Gourdon and C. Joachim, Nano Lett., 2005, 5, 859.

8 F. Rosei, M. Schunack, P. Jiang, A. Gourdon, E. Lægsgaard, I. Stensgaard, C. Joachim and F. Besenbacher, Science, $2002,296,328$.

9 L. Gross, K. H. Rieder, F. Moresco, S. M. Stojkovic, A. Gourdon and C. Joachim, Nat. Mater., 2005, 4, 892.

10 R. Barattin and A. Gourdon, Eur. J. Org. Chem., 2009, 1022.

11 G. M. Whitesides, E. E. Simanek, J. P. Mathias, C. T. Seto, D. N. Chin, M. Mammen and D. M. Gordon, Acc. Chem. Res., $1995,28,37$.

12 H. J. van Manen, V. Paraschiv, J. J. Garcia-Lopez, H. Schonherr, S. Zapotoczny, G. J. Vancso, M. Crego-Calama and D. N. Reinhoudt, Nano Lett., $2004,4,441$.

13 J. A. Theobald, N. S. Oxtoby, M. A. Phillips, N. R. Champness and P. H. Beton, Nature, 2003, 424, 1029.

14 W. Xu, M. D. Dong, H. Gersen, E. Rauls, S. Vazquez-Campos, M. Crego-Calama, D. N. Reinhoudt, I. Stensgaard, E. Lægsgaard, T. R. Linderoth and F. Besenbacher, Small, 2007, 3, 854.

15 M. Ruiz-Osés, N. González-Lakunza, I. Silanes, A. Gourdon, A. Arnau and J. E. Ortega, J. Phys. Chem. B, 2006,110, L25573.

16 M. E. Canas-Ventura, W. Xiao, D. Wasserfallen, K. Mullen, H. Brune, J. V. Barth and R. Fasel, Angew. Chem. Int. Ed., $2007,46,1814$.

17 M. Yu, N. Kalashnyk, R. Barattin, Y. Benjalal, M. Hliwa, X. Bouju, A. Gourdon, C. Joachim, E. Lægsgaard, F. Besenbacher and T. R. Linderoth, Chem. Commun., 2010, 46, 5545.

18 M. Yu, N. Kalashnyk, W. Xu, R. Barattin, Y. Benjalal, E. Lægsgaard, I. Stensgaard, M. Hliwa, X. Bouju, A. Gourdon, C. Joachim, F. Besenbacher and , T. R. Linderoth, ACS Nano, 2010, 4, 4097.

19 T. Zambelli, S. Goudeau, J. Lagoute, A. Gourdon, X. Bouju and S. Gauthier, ChemPhysChem, 2006, 7, 1917. 